\title{
NUVEM DE PONTOS DO MUSEU PAULISTA DA USP: \\ WORKSHOP ENTRE O DIAPREM (UNIVERSIDADE DE FERRARA) E A FAU-USP
}

BEATRIZ MUGAYAR KÜHL, UNIVERSIDADE DE SÃO PAULO, SÃO PAULO,

SÃO PAULO, BRASIL.

É arquiteta formada pela Faculdade de Arquitetura e Urbanismo da Universidade de São Paulo (FAU-USP), com especialização e mestrado em preservação de bens culturais pela Katholieke Universiteit Leuven e doutorado pela FAU-USP, onde é professora titular do Departamento de História da Arquitetura e Estética do projeto.

E-mail: bmk@usp.br

MARCELLO BALZANI, UNIVERSITÀ DEGLI STUDI DI FERRARA, FERRARA, ITÁLIA. Arquiteto, diretor e responsável científico pelo Development of Integrated Automatic Procedures for Restoration of Monuments (DIAPReM), do Departamento de Arquitetura da Universidade de Ferrara, onde é professor titular.

E-mail: marcello.balzani@unife.it

RENATA CIMA CAMPIOTTO, UNIVERSIDADE DE SÃO PAULO, SÃO PAULO,

SÃO PAULO, BRASIL.

Arquiteta formada pela Faculdade de Arquitetura e Urbanismo da Universidade de São Paulo (FAU-USP) e estudante de doutorado do Programa de Pós-Graduação em Arquitetura e Urbanismo da mesma instituição.

E-mail: renata.campiotto@usp.br

RECEBIDO

03/12/2018

DOI

APROVADO

http://dx.doi.org/10.11606/issn.1980-4466.v14i27p310-323

$07 / 12 / 2018$ 


\section{NUVEM DE PONTOS DO MUSEU PAULISTA DA USP: WORKSHOP ENTRE O DIAPREM (UNIVERSIDADE DE FERRARA) E A FAU-USP \\ BEATRIZ MUGAYAR KÜHL, MARCELLO BALZANI, RENATA CIMA CAMPIOTTO}

\section{RESUMO}

Esta notícia apresenta atividades de cooperação entre o laboratório de pesquisa da Universidade de Ferrara - Development of Integrated Automatic Procedures for Restoration of Monuments (DIAPReM) - e a Faculdade de Arquitetura e Urbanismo da Universidade de São Paulo (FAU-USP) sobre o Museu Paulista da USP. As atividades, realizadas na sede da própria FAU-USP, na Cidade Universitária, e no Museu Paulista da USP entre 29 de agosto e 8 de setembro de 2018, foram estruturadas em: reuniões de trabalho; apresentações voltadas ao público em geral; e seminário voltado à formação de equipe de estudantes da FAU-USP para trabalhar com a nuvem de pontos do Museu Paulista. A nuvem foi obtida a partir do escaneamento a laser em três dimensões de todo o edifício do museu, executado pelo DIAPReM, que permite obter a geometria precisa da construção e a morfologia de degradações. Todo o processo foi acompanhado de discussões de questões teóricas e metodológicas, análises críticas do processo de escaneamento, suas características, resultados, aplicações, limites e potencialidades.

\section{PALAVRAS-CHAVE}

Escaneamento $3 \mathrm{D}$ laser. Levantamento tridimensional. Patrimônio arquitetônico. 


\section{POINT CLOUD OF THE MUSEU PAULISTA DA USP: WORKSHOP BETWEEN DIAPREM (UNIVERSITY OF FERRARA) AND FAU-USP \\ BEATRIZ MUGAYAR KÜHL, MARCELLO BALZANI, RENATA CIMA CAMPIOTTO}

\section{ABSTRACT}

This text presents the joint activities between the research laboratory of the University of Ferrara - Development of Integrated Automatic Procedures for Restoration of Monuments (DIAPReM) and FAU-USP (Faculdade de Arquitetura e Urbanismo da Universidade de São Paulo) regarding the Museu Paulista da USP. The activities were carried out at FAU-USP's own headquarters, in the Campus, and at Museu Paulista between August 29 and September 8, 2018, being structured as: work meetings; presentations aimed at the general public; and seminar focused on the formation of FAU-USP team to work with the point cloud of Museu Paulista. The cloud was acquired by means of $3 \mathrm{D}$ laser scanning of the whole museum made with DIAPReM, allowing to obtain the precise geometry of the building and the morphology of its degradations. The whole process was associated with discussion on theoretical and methodological matters, critical analyzes of the scanning process, its characteristics, results, applications, limits and potential.

\section{KEYWORDS}

3D laser scanning. Three-dimensional survey. Architecture heritage. 


\section{INTRODUÇÃO}

Como parte das atividades ligadas ao Grupo de Trabalho Museu Paulista 2022 (GTMP2022), criado pelo reitor da Universidade de São Paulo (USP), foi dada continuidade aos trabalhos de colaboração com o laboratório de pesquisa da Universidade de Ferrara - Development of Integrated Automatic Procedures for Restoration of Monuments (DIAPReM) -, cujo diretor é Marcello Balzani, também responsável científico pelo laboratório e pelo projeto. A USP, pelo Centro de Preservação Cultural (CPC-USP), tem acordo de cooperação acadêmica com o DIAPReM desde 2015, além de pesquisas conjuntas em andamento com a Faculdade de Arquitetura e Urbanismo (FAU-USP), coordenadas por Beatriz Mugayar Kühl. O coordenador do projeto por parte do DIAPReM foi Luca Rossato.

O intuito da colaboração com o GTMP2022 foi elaborar o escaneamento a laser em três dimensões de todo o edifício do Museu Paulista, de modo a obter a geometria precisa da construção, que permite controlar suas deformações e, ainda, a morfologia da degradação de suas superfícies. A aquisição da nuvem de pontos foi realizada em trabalho de campo em agosto de 2017, com coordenação de Guido Galvani e participação de Daniele Sasso. Em seguida, o material foi tratado no Laboratório do DIAPReM, em Ferrara, e enviado em fases à USP, até a entrega final e o encerramento dos trabalhos, entre agosto e setembro de 2018. 
Como parte do processo, foi prevista a realização de um seminário em São Paulo, tema desta notícia, com a presença de três membros da equipe de Ferrara: o diretor do laboratório, Marcello Balzani, e os professores Federica Maietti e Guido Galvani. As atividades foram estruturadas em reuniões de trabalho, apresentações voltadas ao público em geral e seminário voltado à formação específica de equipe de estudantes da FAU-USP para trabalhar com a nuvem de pontos do Museu Paulista.

As apresentações abertas ao público tiveram por objetivo: discutir as características metodológicas do levantamento arquitetônico integrado e da tecnologia do escaneamento a laser em três dimensões; analisar casos de estudo; apresentar conceitos básicos de nuvem de pontos; mostrar tipos de rendering possíveis e como é feita a geração de plantas, cortes e maquetes; exemplificar a análise macroscópica das morfologias de degradação; apresentar conceitos teóricos relacionados ao levantamento integrado nas estratégias de conservação; e discutir os resultados obtidos a partir da nuvem de pontos do Museu Paulista (Figura 1). Desse modo, puderam usufruir da análise dos resultados não apenas os estudantes de graduação e pós-graduação da FAU-USP, mas também profissionais da área, muitos ligados a órgãos públicos de preservação, e interessados em geral.

FIGURA 1

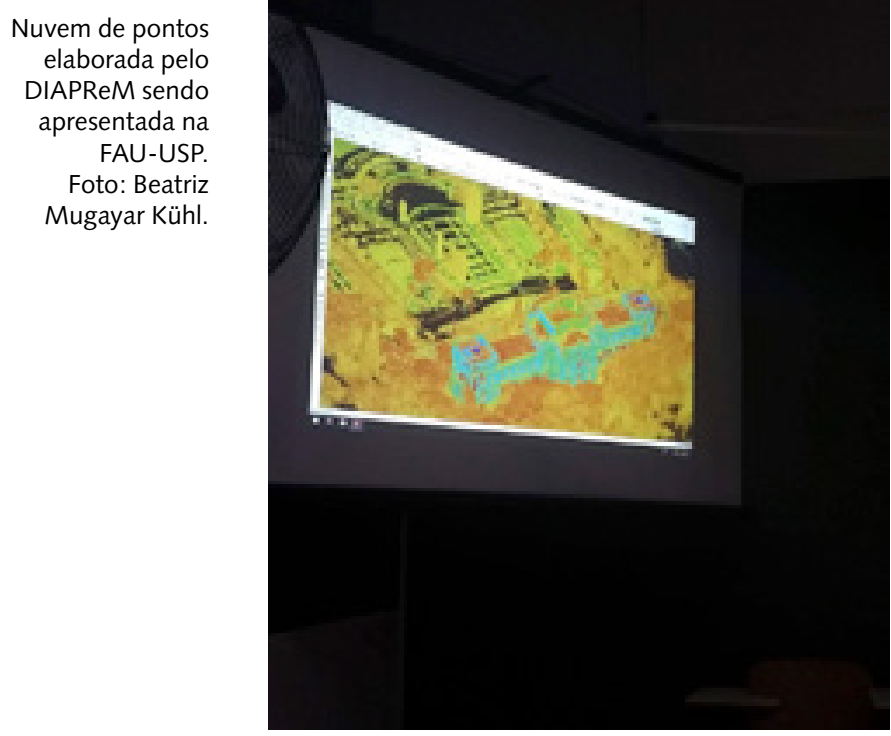


O conjunto das atividades foi estratégico tanto para debater metodologia e resultados obtidos para o Museu Paulista com o público em geral quanto para formar a equipe local para continuar o trabalho com a nuvem de pontos. As atividades foram estruturadas segundo o seguinte cronograma:

29 de agosto (quarta-feira)

Reunião de organização, discussão da logística

Verificação dos equipamentos e das salas a serem utilizados

Entrega da nuvem de pontos global do Museu Paulista

30 de agosto (quinta-feira)

9 h às 12h30: seminário sobre a nuvem de pontos para a equipe FAU-USP.

14h3o às 18h3o: apresentação aberta ao público: "Escaneamento a laser em três dimensões: teoria e método".

31 de agosto (sexta-feira)

9h às 12h30: seminário sobre a nuvem de pontos para a equipe FAU-USP.

14h30 às 18h30: seminário sobre a nuvem de pontos para a equipe FAU-USP. Preparação para o trabalho de campo. Diagnóstico.

$1^{\circ}$ de setembro (sábado)

9h3o às 18h30: trabalho de campo no Museu Paulista para a equipe FAU-USP: visita ao edifício; verificação das fachadas externas, apontando principais patologias; realização de eidotipo (eidos = aspecto/ forma + tipo), representação a mão livre do edifício, devidamente trabalhado em suas proporções; registro e análise das patologias de trechos das fachadas.

3 de setembro (segunda-feira)

9h às 12h30: seminário sobre a nuvem de pontos para a equipe FAU-USP.

14h3o às 18h3o: seminário sobre a nuvem de pontos para a equipe FAU-USP. 
4 de setembro (terça-feira)

9h3o às 12h3o: apresentação aberta ao público: Diagnóstico integrado. Teoria e método.

14h30 às 18h30: seminário sobre a nuvem de pontos para a equipe FAU-USP. Conclusões.

5 de setembro (quarta-feira)

9h3o às 12h3o: reunião de trabalho entre a equipe DIAPReM e Beatriz Mugayar Kühl. Balanços.

14h3o às 17h30: apresentação pública dos resultados da nuvem de pontos do Museu Paulista. Local: Museu Paulista, auditório da Avenida Nazaré.

6 de setembro (quinta-feira)

9h3o às 12h30: reunião de trabalho entre a equipe FAU-USP e DIAPReM.

Apresentação pública da nuvem de pontos no Circolo Italiano. 7 de setembro (sexta-feira)

9h3o: participação da Cerimônia de Abertura do "Museu do Ipiranga em Festa", organizada no Museu Paulista, a convite da Reitoria da USP.

8 de setembro (sábado)

12h: reunião entre professores do DIAPReM e Beatriz Mugayar Kühl para um balanço dos trabalhos e estruturação das próximas etapas.

\section{SOBRE O TREINAMENTO OFERECIDO AOS ESTUDANTES}

$O$ treinamento oferecido pela equipe do DIAPReM foi iniciado pela exposição de princípios básicos do levantamento métrico-arquitetônico, seus objetivos, métodos e resultados. Outro ponto abordado na primeira fase tratou da tecnologia do levantamento por varredura a laser, ou seja, a forma como o aparelho opera, os modos de controle de acordo com os objetivos da operação e, portanto, a lógica de seu funcionamento.

Em seguida, a abordagem do treinamento voltou-se ao processo de captura de dados, que deve ser coerente com a geometria do objeto de estudo, de seu entorno e das condições ambientais existentes. 
O professor Guido Galvani, responsável por essa etapa do treinamento, utilizou a nuvem de pontos já processada do edifício-monumento do Museu Paulista para ilustrar esses conceitos, sobretudo aqueles relativos aos dados obtidos a partir do levantamento que, posteriormente, serviram de base para tratar das possibilidades de diagnóstico do estado de conservação das superfícies do edifício.

Após a primeira etapa de formação, de caráter teórico, passou-se ao treinamento com o software de processamento e manipulação da nuvem de pontos, o Cyclone. Esse programa, desenvolvido pela Leica Geosystems, permite não somente a integração e a limpeza dos dados capturados durante o escaneamento a laser, mas também a elaboração de produtos gráficos. A partir dos comandos existentes no Cyclone, é possível exportar ortofotos de plantas, cortes, elevações e detalhes, que podem ser exportadas também para o software AutoCAD e atuar como levantamento métrico de grande precisão. Sobre esse tópico, foi discutida a questão dos erros possíveis e toleráveis, inerentes à operação do levantamento.

A partir da análise crítica em relação ao método e aos resultados, foram então discutidas, pela professora Federica Maietti, as formas de uso dos produtos do levantamento para o auxílio no diagnóstico integrado do estado de conservação das superfícies. Para isso, no sábado, $1^{\circ}$ de setembro, foi realizado um trabalho de campo. A partir de desenhos à mão livre realizados in loco, essenciais para compreender a implantação, a geometria, a proporção e a composição volumétrica do edifício e de seu entorno, deu-se início à leitura pormenorizada de seções das fachadas (Figuras 2 e 3). Buscou-se comparar, entre imagens extraídas da nuvem de pontos e o edifício em si, indícios de danos e manifestações patológicas presentes nas superfícies. 


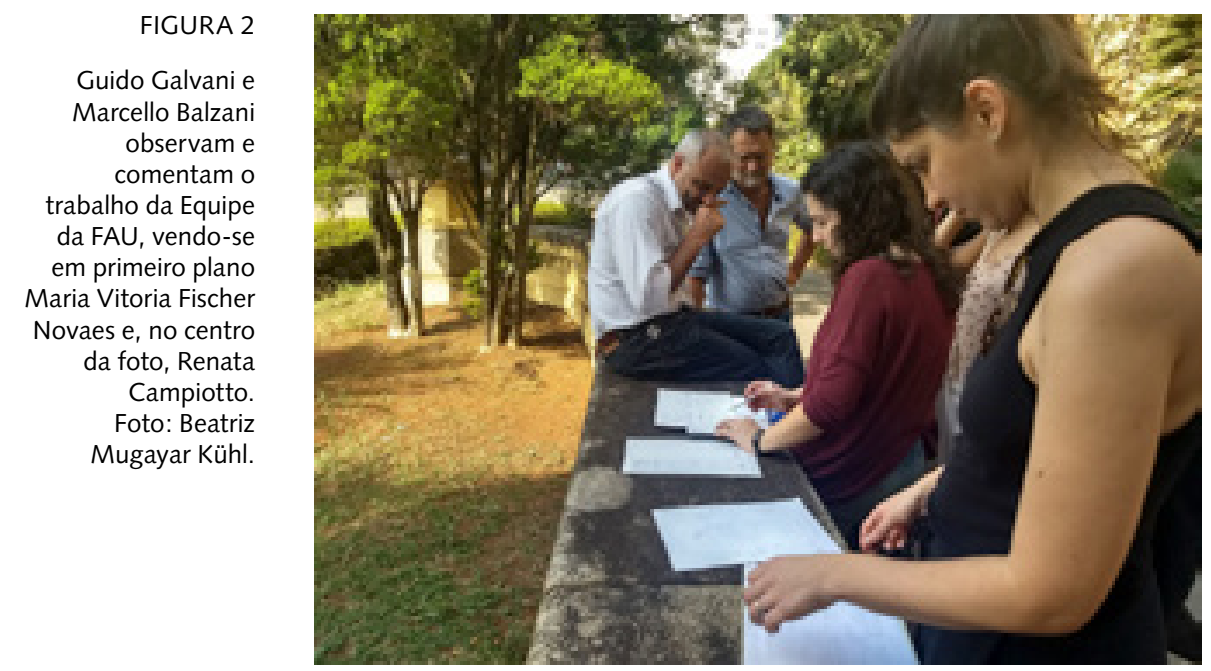

FIGURA 3

Guido Galvani,

Marcello Balzani

e Federica Maiett

examinam os

trabalhos, sendo

observados por

Stephanie Luna

Galdino. Foto: Beatriz

Mugayar Kühl.

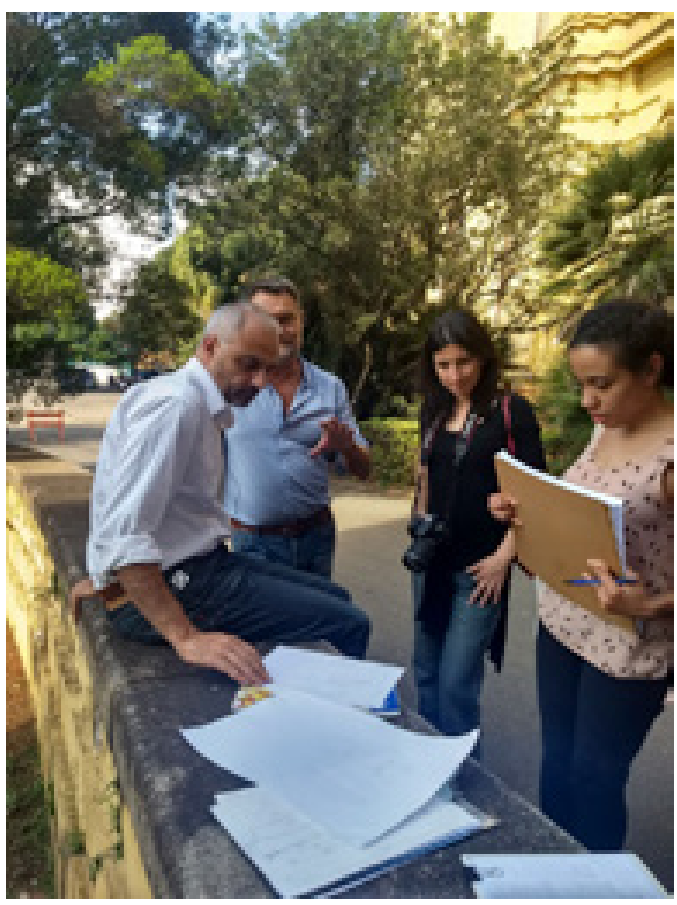

Os demais dias de treinamento tiveram como foco a extração de imagens e a manipulação dos dados - mais precisamente, a forma como a mudança da escala dos parâmetros de refletância, dado obtido no escaneamento a laser, poderia indicar diferenças de comportamento dos materiais das superfícies do museu. O conjunto de dados obtidos pelo escaneamento fornece bases precisas para o desenho e a análise do edifício. 
O encerramento das atividades de formação contou com um seminário, realizado no dia 4 de setembro de 2018 e aberto ao público, em que foi apresentado o balanço de todas as operações - levantamento de campo, processamento dos dados, manipulação da nuvem e resultados obtidos. Também foi discutida a importância e a relevância dessa tecnologia para aplicação em objetos complexos, como é o caso do edifício do Museu Paulista.

\section{ALGUMAS CONSIDERAÇÕES SOBRE O ESCANEAMENTO A LASER EM TRÊS DIMENSÕES}

$\mathrm{Na}$ fase de decisão do projeto de cooperação com a Universidade de Ferrara, assim como na estruturação do trabalho a ser desenvolvido no Museu Paulista, e durante todo o processo de formação acima descrito, foram muito discutidas as características do escaneamento a laser por todos os membros do DIAPReM.

É essencial conhecer as características do objeto a ser levantado para projetar o levantamento, pois podem ocorrer variações significativas em função das características da obra e também do grau de precisão adequado para o trabalho a ser desenvolvido. Não é necessário, nem pertinente, por exemplo, realizar um escaneamento a laser para fazer a reforma de um apartamento residencial que não apresenta maiores dificuldades; por outro lado, o método é essencial para o levantamento rigoroso de edifícios complexos que apresentam problemas intrincados, caso do Museu Paulista. O escaneamento permite obter a geometria da obra com precisão, possibilitando a análise aprofundada de suas características e de eventuais deformações. Desse modo, auxilia em diversas frentes de trabalho, como o diagnóstico estrutural, pois faculta examinar com precisão desníveis e desaprumos, assim como viabiliza, por exemplo, um estudo comparativo preciso de capitéis, balaústres, portas etc.

Além da capacitação da equipe da FAU, foi também estabelecida uma colaboração com o escritório Hereñu + Ferroni, vencedor do concurso de projeto para o museu, de modo a oferecer dados e elementos para o desenvolvimento do projeto executivo.

Como sempre enfatiza Marcello Balzani, o escaneamento é primordial para conservar a memória geométrica de dada construção num determinado momento; ou seja, é base de referência importante para 
controlar o comportamento da obra ao longo do tempo. Ter a nuvem de pontos do museu no estado atual é essencial para documentar seu estado preciso e real - e não a partir de retificações e dimensionamentos abstratos - antes da obra. Pretende-se também, se possível, realizar alguns escaneamentos durante a obra e, depois, programar outra campanha de escaneamento no futuro, com as obras terminadas, o que permitirá realizar análises comparativas de antes, durante e depois da intervenção, propiciando o exame pormenorizado do comportamento do edifício. O escaneamento pode ainda ser articulado a outros métodos (a exemplo de espectrofotometria, fluorescência UV etc.), que facultam um diagnóstico integrado - tema enfrentado sobretudo por Federica Maietti nas atividades na FAU.

Algo que já era explícito na proposta de trabalho e que foi sempre enfatizado nas apresentações públicas é que o escaneamento a laser em três dimensões é um método utilíssimo, mas extremamente complexo. Todas as etapas devem ser preparadas e executadas de forma rigorosa, o que exige profundo conhecimento do método, do instrumento a ser utilizado e do objeto a ser escaneado. O escaneamento, além de dever ser projetado de modo consciente, deve ser executado com extremo rigor - respeitando a precisão do aparelho, as necessárias sobreposições entre os escaneamentos das diversas estações etc. - e, depois, os dados obtidos devem ser tratados com igual diligência para se obter uma nuvem de fato precisa. Ou seja, são necessárias muitas horas de trabalho de profissionais altamente gabaritados. A equipe da FAU teve noções básicas do procedimento como um todo e passou por treinamento para obter produtos a partir da nuvem de pontos (Figuras 4, 5, 6 e 7).

\footnotetext{
FIGURAS 4 E 5

Equipe da FAU trabalhando na nuvem. Da esquerda para a direita: Maria Vitória Fischer Novaes e Stephanie

Luna Galdino;

Renata Campiotto e Stephanie Luna Galdino. Foto: Renata Campiotto (Figura 4) e Maria Vitória Fischer (Figura 5).
}
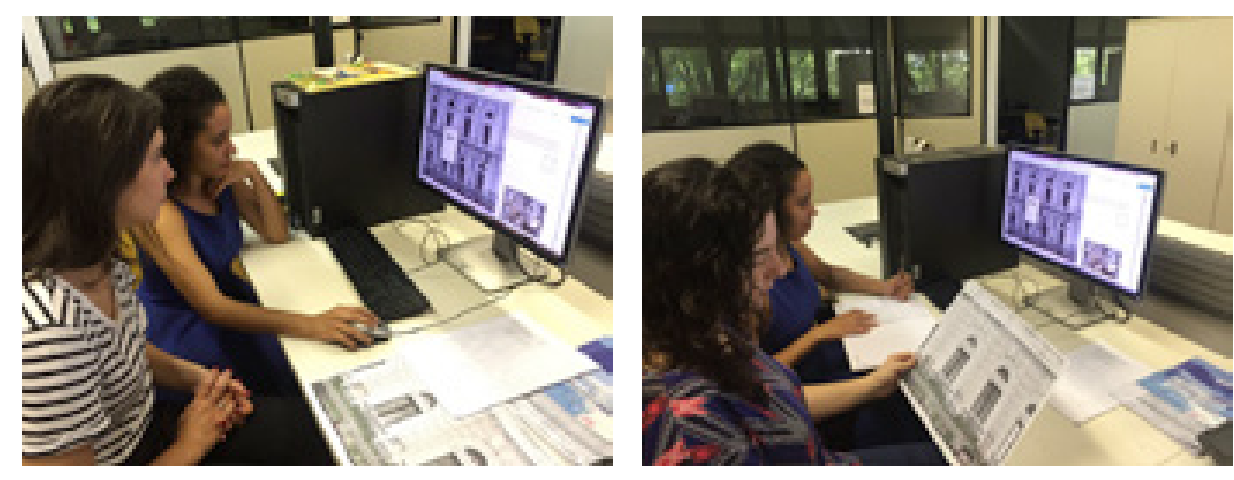
FIGURA 6

Vista da fachada norte do Museu Paulista trabalhada pela equipe da FAU. Fonte: obtida a partir da nuvem elaborada pelo DIAPReM.

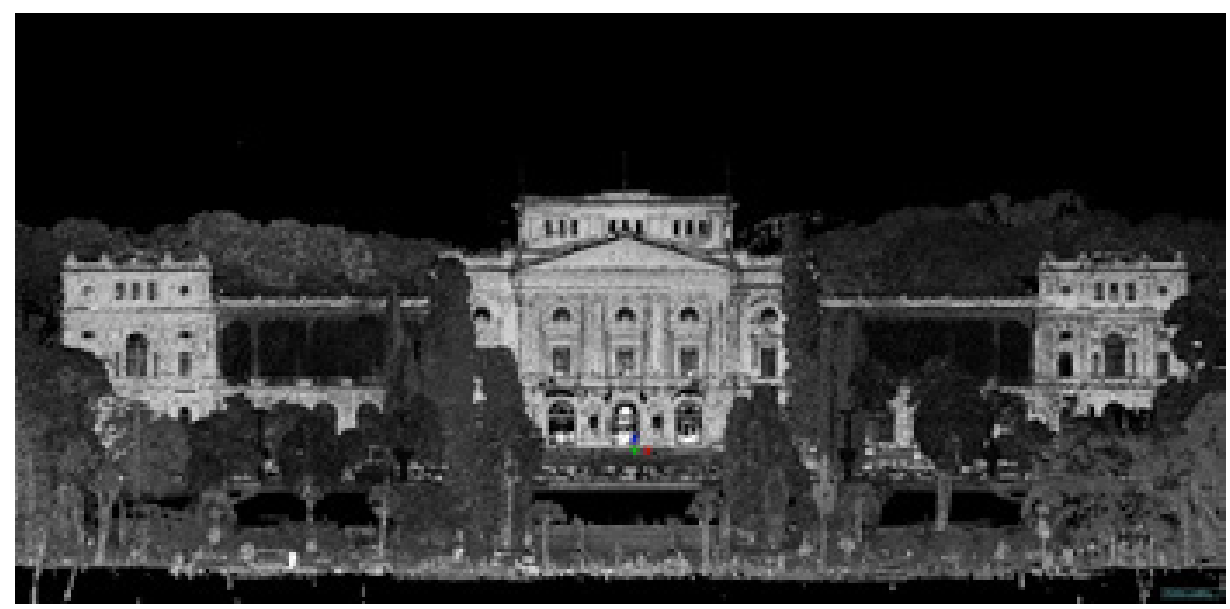

FIGURA 7

Pormenor da fachada sul obtido por Renata Campiotto. Fonte: Obtida a partir da nuvem elaborada pelo DIAPReM.

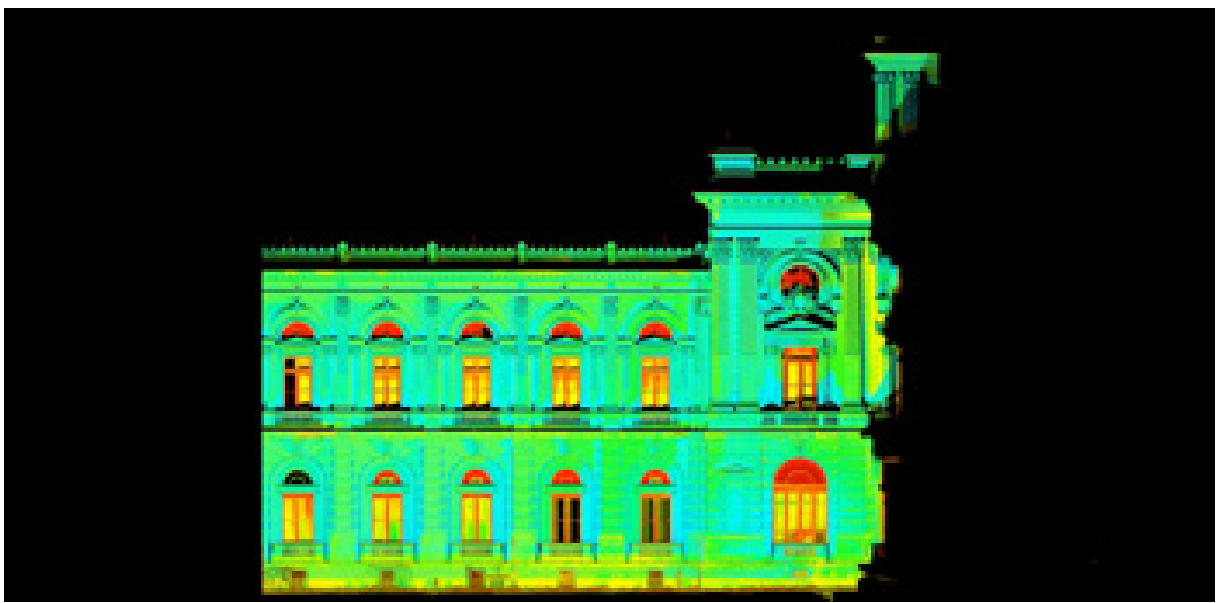

Dada a complexidade do processo, a equipe da FAU ainda não tem autonomia para projetar um escaneamento, realizá-lo e tratar a nuvem de pontos - algo que exige mais tempo de treinamento e experiências sucessivas realizadas sob supervisão de profissionais capacitados. Deve-se lembrar que membros da equipe de Ferrara trabalham no campo de levantamentos com métodos avançados há mais de duas décadas 1 . O intuito do workshop na FAU-USP era informar, sensibilizar sobre a complexidade dos procedimentos e oferecer capacitação básica para extrair alguns elementos e dados da nuvem.

1. Para visualizar os trabalhos realizados pelo laboratório, acessar a página do DIAPReM: http:// www.diaprem.unife.it/archivio-progetti. 
Algo que se deve ter sempre em mente é que uma nuvem mal executada tem uma aparência tão "bonita" quanto uma nuvem executada com rigor em todas as etapas. Será, porém, extremamente imprecisa e, portanto, inutilizável para fins de restauro e para qualquer trabalho que exija precisão geométrica, que só é alcançada numa nuvem trabalhada de modo rigoroso. Ou seja, se não se souber exatamente como o levantamento foi projetado e executado e, depois, como os dados foram tratados, a nuvem poderá ter, superficialmente, uma "aparência" adequada, mas será imprecisa e inapropriada para análise, e - o que é mais grave -, sendo uma nuvem malfeita, pode induzir a erros de interpretação.

Convém também esclarecer dois equívocos recorrentes e diametralmente opostos em relação ao escaneamento a laser: há os que acreditam que a nuvem dá todas as respostas sozinha; e os que creem ser um método custoso, que não serve para nada. O escaneamento a laser em três dimensões não é nem uma coisa, nem outra - algo muito discutido pelos representantes do DIAPReM e que é possível verificar pela discussão precedente.

Como Marcello Balzani reitera constantemente, sozinha a nuvem não dá respostas; essa técnica não fala espontaneamente, e, portanto, a nuvem deve ser projetada, tratada e indagada com acuidade. Ou seja, a inteligência está nos operadores. Conhecendo as características e potencialidades da nuvem, é possível alcançar respostas que não seriam obtidas de outra forma, mas para isso é necessário saber fazer as perguntas. Essa capacidade vem do conhecimento dos campos envolvidos com a arquitetura e da restauração arquitetônica.

Se usada inteligentemente, além de oferecer bases geométricas num grau de precisão impossível de ser atingido por instrumentos tradicionais, a nuvem pode ser associada a outros procedimentos e contribuir de modo decisivo em diagnósticos.

Equipe técnica: DIAPReM

Diretor e responsável científico: Marcello Balzani

Coordenador do projeto: Luca Rossato

Levantamento diagnóstico: Federica Maietti

Técnicos de levantamento: Guido Galvani (coordenador)

e Daniele Felice Sasso 


\section{FAU-USP}

Coordenação do projeto na FAU-USP: Beatriz Mugayar Kühl

Equipe técnica: FAU-USP

Coordenação da equipe: Renata Cima Campiotto

Maria Vitoria Fischer Novaes

Stephanie Luna Galdino

Demais participantes da equipe FAU:

Eduardo Ribeiro

Jessica Mari Hanao

Cartherine Calognomos

Financiamento: Fundação de Apoio à Universidade de São Paulo (FUSP)

Organização: FAU-USP, Museu Paulista, DIAPReM 